\title{
EMPIRICALLY INFORMED SOUND SYNTHESIS APPLICATION FOR ENHANCING THE PERCEPTION OF EXPRESSIVE ROBOTIC MOVEMENT
}

\author{
Jon Bellona \\ University of Virginia \\ Department of Music \\ Charlottesville, VA, USA \\ jpbellona@virginia.edu \\ Luke Dahl \\ University of Virginia
Department of Music
Charlottesville, VA, USA
lukedahl@virginia.edu
}

ABSTRACT

Since people often communicate internal states and intentions through movement, robots can better interact with humans if they too can modify their movements to communicate changing state. These movements, which may be seen as supplementary to those required for workspace tasks, may be termed "expressive." However, robot hardware, which cannot recreate the same range of dynamics as human limbs, often limit expressive capacity. One solution is to augment expressive robotic movement with expressive sound.

To that end, this paper presents an application for synthesizing sounds that match various movement qualities. Its design is based on an empirical study analyzing sound and movement qualities, where movement qualities are parametrized according to Laban's Effort System. Our results suggests a number of correspondences between movement qualities and sound qualities. These correspondences are presented here and discussed within the context of designing movement-quality-to-sound-quality mappings in our sound synthesis application. This application will be used in future work testing user perceptions of expressive movements with synchronous sounds.

\section{INTRODUCTION}

Humans often communicate intentions and affective states through qualitative aspects of their body movement. For example, the same hand movement can elicit two different responses - warmth, as when one points to a friend on the street, or aggression, as when one points out the accused in a police lineup. The difference is in the context and how the gesture is executed.

(c) (1) (5) This work is licensed under Creative Commons Attribution Non Commercial 4.0 International License. The full terms of the License are available at http://creativecommons.org/licenses/by-nc/4.0

\author{
Lin Bai
}

\author{
University of Virginia \\ Electrical and Computer Engineering \\ Charlottesville, VA, USA \\ lb7ssevirginia.edu \\ Amy LaViers \\ University of Illinois \\ Mechanical Science and Engineering \\ Champaign-Urbana, IL, USA \\ alaviersdilinois.edu
}

https://doi.org/10.21785/icad2017.049

Robots in human-facing roles could more effectively interact with humans if they too could express various intentions through their movement. Ongoing research is being conducted with the goal of endowing robots with the ability to express different qualities in their movement $[1,2]$. However, the physical limitations of specific robotic platforms can reduce a robot's ability to sufficiently express differences in movement qualities [3].

Humans also communicate intentions and emotions sonically through non-verbal aspects of their vocalizations. For example, an angry person might modulate the loudness and resonance of their voice, which mirrors shifts in body language and emotion. Thus, by endowing robots with expressive sound we may improve people's perception of varying qualities in robotic movement. The question then becomes, how does one design sounds to accompany expressive movements with perceptual cohesion?

In this paper we present a quantitative, empirically-based sound design approach and a synthesis application for generating sounds to accompany various movement types. We summarize a study we conducted [4], whose results suggest a number of correspondences between movement qualities and sound qualities. And we describe how these correspondences are used to control sound synthesis parameters in our application.

In our study we asked experienced musicians to vocalize sounds to animations of a simple movement. Different versions of this movement were generated by varying the Effort Factors proposed by movement theorist Rudolf Laban (see Section 2.1). The musicians' vocalizations were recorded and then analyzed by manually applying qualitative labels to each sound, and by performing a quantitative signal analysis using techniques from MIRtoolbox [5]. Our goal was to discern general trends in how sounds differ as movement quality varies.

We applied the study results to a sound synthesis application in which we dynamically modify sound quality parameters according to changes in movement quality. We control our sound synthesis with the same movement quality parameters that are used to generate movement trajectories for robotic movement (as described in [3]). 


\section{BACKGROUND}

A number of studies have been conducted on movement sonification. In music, studies of body movements of acoustic musicians by [6] show correlations of qualitative movement related to structural components (e.g. tempo) within musical works. Another study correlates expressive movement to musical phrasing by tracing movement shapes of nine pianists [7]. Our work moves the other way in taking movement qualities and mapping these onto their respective parameters of sound qualities in a sound synthesis application.

Movement sonification studies in athletic tasks demonstrate acute human perception of changes in timing with movement sonification [8] [9]. Instead of sonifying repetitive sequenced patterns of movements, our sound design focuses on generating sounds based upon short, singular movements. Effenberg et al. [10] used a direct mapping of "kinematic and dynamic motion parameters to electronic sounds" to help improve motor learning in sports. While we also integrate multi-modal perception into our work, we are interested in designing sounds that correspond to basic movement qualities instead of designing for specific sequenced movement structures used in specific tasks.

Similarly, [11] and [12] measured kinematic features of movement and transformed these features into sound. And [13] presents a real-time sonification framework for all common MIDI environments based upon acceleration and orientation data from inertial sensors. Our sound synthesis integrates Laban Effort Factors, which describe how a movement is performed, with the aim to improve the perception of expressive robotic movement.

Generating robotic movement by mapping the components of Laban's Effort system to weighted parameters in an optimal control problem builds on [14]. Our sound synthesis application parameters leverage sound qualities corresponding to movement qualities from an analysis of vocalizations of movement by [4]. These movement to sound quality correspondences will be discussed further in Section 3. The approach toward generating sounds for preexisting movements is similar to work in [15] in that they use Laban Effort Factors to organize movement; however, their participants train a system with movement data in order to generate sound from dancers' movements in real time. Our work aims to generate sound from movement quality parameters.

Lastly, we acknowledge a rich history of sound mappings for sonification purposes [16]. Sound design is a skilled art. Our goal isn't musical sound design, but rather to communicate intent and affective state with sound along with movement based upon their perceptual links. Rather than using aesthetics to drive mapping choices as seen in [17] and [18], our mapping choices are instead based upon quantitative signal analyses and qualitative results (see Sections 3.1 and 3.2).

\subsection{Describing Movement Quality}

Our synthesis framework, as well as the algorithms we use for generating robotic movement [3], are controlled by parameters based on the Effort system defined by Rudolf Laban $[19,20]$ to qualitatively specify the ways in which a movement may vary. In this system, movements are characterized using four Effort Factors: Space Effort, Time Effort, Weight Effort, and Flow Effort. We capitalize these terms to avoid possible confusion with other notions of these terms.

Space Effort describes the attitude toward the environment of
Table 1: Laban's Eight Basic Effort Actions

\begin{tabular}{|c|ccc|}
\hline Movement & Time & Space & Weight \\
\hline Gliding & Sustained & Direct & Light \\
Pressing & Sustained & Direct & Strong \\
Floating & Sustained & Indirect & Light \\
Wringing & Sustained & Indirect & Strong \\
Dabbing & Sudden & Direct & Light \\
Thrusting & Sudden & Direct & Strong \\
Flicking & Sudden & Indirect & Light \\
Slashing & Sudden & Indirect & Strong \\
\hline
\end{tabular}

a movement. A movement can be Direct (as in throwing a boxing jab), or Indirect (as in shaking out a rock-filled boot). Time Effort describes the attitude towards initiation and completion of a movement. A movement can be Sudden (as in pulling your hand away from a hot stove) or Sustained (as in pushing against a grand piano). Weight Effort describes the attitude towards the mover's mass. A movement may be Strong (as in sprinting a 100 meter dash) or Light (as in touching a baby's cheek). Flow Effort describes the progression of a series of movements. We do not use Flow Effort in our sound application, since the movements analyzed for sound synthesis may all be considered singular movements.

When paired in all combinations, the three Effort Factors that we employ - Space Effort, Weight Effort, and Time Effort - form Laban's eight Basic Effort Actions (BEAs), as shown in Table 1. The BEAs are Dabbing, Flicking, Floating, Gliding, Pressing, Slashing, Thrusting, and Wringing. These eight actions constitute a set of basic movements whose qualities people can understand by drawing on their own experiences [20].

\section{A STUDY ON SOUND MOVEMENT CORRESPONDENCES}

This paper documents an application to generate sounds based upon correspondences between movement and sound, so that we may further study the perception of endowing expressive robotic movement with expressively coherent sound. In order to search for these correspondences we conducted an initial study, whose details are presented in [4], and which we summarize here.

We created animations of a stick figure performing a simple movement with the qualities of each of the eight BEAs. The figure moves from a pose where the hands are near the center line, to a pose where the arms are extended to the sides, and then returns to the first pose. Each movement was four seconds long. The qualitative variations in the movement trajectories are created by changing three parameters of a control algorithm described in [3] and [14]. These parameters correspond to the Laban Effort Factors of Space, Weight, and Time.

We presented these animations to seven musicians (graduate students and professional musicians, all with significant improvisation experience), along with the BEA label, and asked them to vocalize a sound that matched the qualities of the movement and label. We recorded these sounds and subjected them to the analyses described in Section 3.1. The BEA labels were necessary because the animations, though generated with different movement qualities, were not different enough for the participants to be able 
to easily distinguish the differences. Indeed, this is part of the motivation for this work.

\subsection{Quantitative and Qualitative Sound Analysis}

We performed two analyses of the recorded vocalizations. In the first, we manually applied qualitative labels to each recording, and in the second we performed signal analysis in order to quantify various sonic qualities.

In the first analysis, each of the four authors listened to and manually applied qualitative labels to each of the 56 recordings ( 7 musicians $\times 8$ BEAs). We chose a set of labels for the attributes of pitch, loudness, and timbre, as well as for the shape of how pitch, loudness, and timbre varied over each sound's duration. The labels we used can be seen in Figure 1. For each attribute, a listener was allowed to apply only one label. In order to look for meaningful sonic differences between movement qualities, we reorganized the label data according to each Effort Factor (Space, Weight, Time), and created histograms to compare the two values of each Factor. For example, in Figure 1 we can compare Strong and Light Weight Efforts, and we see that the amplitude of sounds for Strong Effort movements are more often labelled 'medium' and 'loud' than are sounds for Light Effort movements.
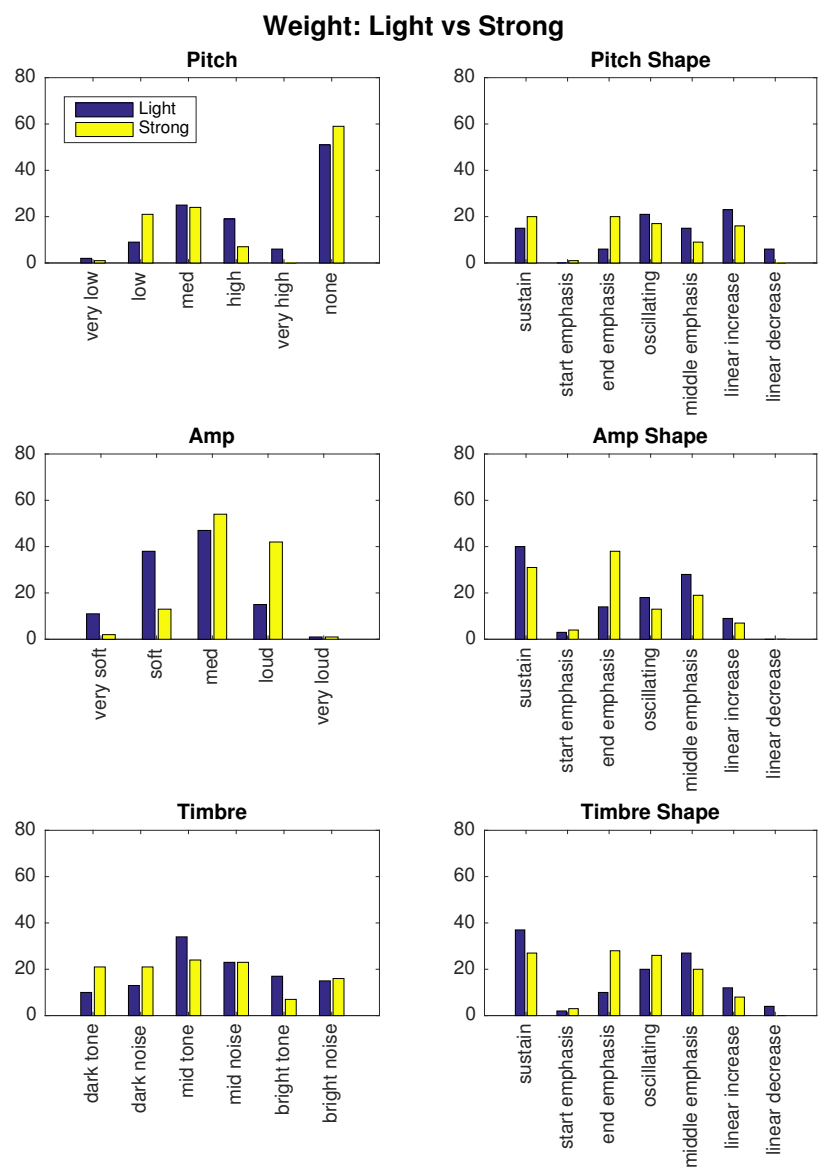

Figure 1: Qualitative label counts for the Effort Factor Weight comparing Strong vs. Light

In the quantitative analysis, we used the MIRtoolbox [5] to extract the following audio features for each recording: amplitude envelope, spectral brightness, spectral centroid, spectral rolloff, spectral flatness, and zero-crossing rate. We calculated the mean value of each feature across each recording, which we call the 'recording mean'. To account for differences in vocal range or performance style, for each participant we subtracted out the mean of each feature taken across all recording means for that participant. We then reorganized the data by Effort Factors, which allows to conduct T-tests to determine if a features varies significantly between the two values of a given Effort Factor. For example, Figure 2 shows that the mean spectral rolloff for movements whose Time Effort is Sudden is higher than for movements whose Time Effort is Sustained, and a T-test confirms this $(t(54)=2.74995, p=0.0081)$.

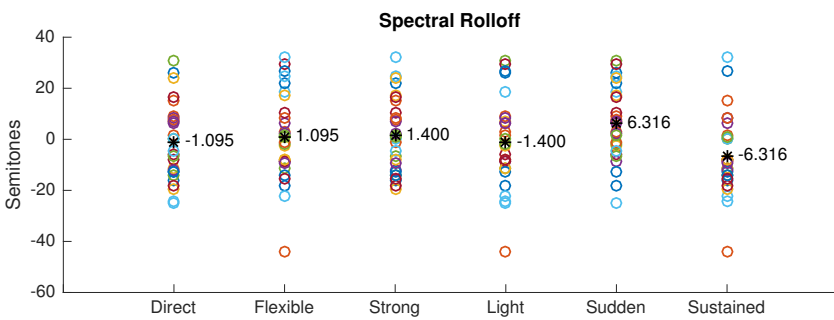

Figure 2: Spectral Rolloff for the Space, Weight, and Time Effort Factors.

\subsection{Sound Movement Correspondence Findings}

Detailed results of our study are described in [4]. Here we summarize the findings, which are used to inform the design of our sound synthesis application. P-values for significant findings from the quantitative analysis are shown in Table 2.

Here we describe the findings for each Effort Factor, and indicate whether they came from the qualitative (qual) or quantitative (quant) analysis.

\section{Time Effort:}

- Sudden movements are associated with brighter sounds, whereas sounds for Sustained movements tend to have darker timbres (quant and qual).

- Sudden movements are associated with noisier sounds, whereas sounds for Sustained movements are more pitched (quant and qual).

- Sounds for Sudden movements tend to have moments of strong emphasis both in amplitude and timbre, whereas sounds for Sustained movements tend to have smooth amplitude envelopes and smoothly varying timbre (quant and qual).

- Sounds for Sudden movements tended to be louder than for Sustained movements (qual).

Weight Effort:

- Strong movements are associated with sounds that are louder and have higher peak amplitudes, whereas sounds for Light movements are quieter and have smaller peak amplitudes (quant and qual).

- Strong movements are associated with darker sounds, whereas Light movements are associated with brighter sounds (qual). 
- Sounds for Strong movements tend to have stronger end emphasis, whereas sounds for Light movements tend to have middle emphasis or were sustained (qual).

Interestingly, we did not find any notable differences when comparing Direct and Flexible Space Effort. This was true for both the qualitative and quantitative analyses. We hypothesize that this may be true because the concept of space does not map very precisely onto the qualities of sounds we studied, and a full discussion is available in [4].

\section{SYNTHESIS APPLICATION}

We created a sound synthesis application based upon correspondences between movement and sound (see Section 3). Sound quality parameters directly correlate to movement quality parameters from the movement trajectory function of [3], so that single robotic movement trajectories may be sonified and recorded; although, the software was designed with the potential to translate multiple movement trajectories into sound.

We built our synthesis application with Max/MSP ${ }^{\mathrm{TM}}$ software. The application was primarily built using native Max 6 objects, but utilizes scaling functions developed by [21]. We chose electronic synthesis over a sample-based approach in order to retain the most parametric control over our designed sounds. Nearly all audio components are informed by our previous study [4], and parameter ranges used to match the results are based in part by active listening and iteration, as well as psychoacoustic phenomena. Next, we will describe the audio circuit before discussing each Effort Factor parameter in more detail.

\subsection{Audio Circuit (Signal Flow)}

The sonification uses a combination of additive and subtractive synthesis techniques. We start by generating pitched sounds with three summed oscillators. A rectangle wave sounds the fundamental frequency, and two sawtooth waves sit an octave above (with one of the sawtooth waves being slightly detuned). The summed oscillators move through a Moog ${ }^{\mathrm{TM}}$ ladder resonant filter, and movement qualities control the fundamental frequency of the sound, resonant frequencies of the filter, and the sound's amplitude envelope (ADSR) (described in section 4.3).

Separately, a white noise generator feeds a state-variable filter with a center frequency of $4.1 \mathrm{kHz}$. Both pitch and noise sounds have an ADSR envelope and duration controls to regulate emphasis peaks of amplitude, and each have separate gain controls. After summing noise and pitch signals, the combined signal splits. One signal is kept 'dry' (no effect) and the other signal feeds a detuned delay (chorus effect) that also spreads in the stereo field. Effected and dry signals are combined in the left and right channels, which feed a low-pass biquad filter. A final gain control determines overall amplitude of the sound. For the full schematic, see Figure 3.

\subsection{User Parameters}

The application interface provides the user with several user parameters, chiefly three main inputs, in the form of Laban Effort Factors: Weight, Time, and Space. These parameter values have a range between $0-1000$, with extremes of each range matching movement qualities labels from Table 1 . The value ranges in the synthesis application match movement quality parameter values used for generating movement trajectories [3].
Because movement trajectories depend on robotic platforms to carry out generated movements, physical differences between platforms may alter a movement's duration. In addition, the shape of the generated movement does not indicate where in the movement the emphasis will occur, only that an emphasis will occur. For example, the impulse of a Sudden movement may occur at the beginning or end of the movement, but both movements may be deemed as Sudden.

Two additional user parameters affect overall duration and time location of peak amplitude emphasis. These two envelope parameters are set manually in order to match singularly generated movement trajectories. For multiple movement recordings, these two user parameters may need to be automatically updated in order to dynamically respond to trajectory inputs. For now, the sound synthesis application has been used to record single movement sounds, and these sounds will be used for a forthcoming study measuring the perception of our quantitative sound design and movement trajectories. The next section will discuss movement qualities and their associative sound controls.

\subsection{Laban Effort Factor Sound Controls}

$$
\text { 4.3.1. Time }
$$

Time Effort values affect amplitude peak and gains for pitched and non-pitched signals as well as overall brightness. These controls correlate to quantitative results of sound qualities shown in Table 2. For amplitude peaks, Sudden Time Effort generates stronger, or emphasized, amplitude peaks and Sustained Effort generates a smooth amplitude envelope curve, void of any peaks. Time Effort simultaneously controls gains for both pitched and non-pitched signals. Sudden sounds tend to be noisier, so that Sudden Effort emphasizes noise signals, with white-noise gain set to OdBFS and electronically pitched tone signal gain set to -12dBFS. Sustained Effort inversely emphasizes pitched signals, with pitched sound signal gain at OdBFS and white-noise sound signal gain at $-12 \mathrm{dBFS}$.

Time Effort also affects overall brightness by controlling a cutoff frequency of a low-pass bi-quad filter. Sudden Effort maintains the high-end of sounds, whereas Sustained Effort rolls off higher frequencies to produce darker sounds. The large roll-off range of $1 \mathrm{kHz}-10 \mathrm{kHz}$ was chosen in order to be perceptible enough for most listeners.

\subsubsection{Weight}

Weight Effort controls the peak amplitude of pitched and nonpitched signals, oscillator frequencies and resonant filter frequency of pitched signals, as well as a fine adjustment control of overall amplitude. For peak amplitude, Weight Effort adds additional peak emphasis to signal envelopes, and the peak envelope parameter stands on the quantitative correlation shown in Table 2. Thus, Weight Effort combines with Time Effort in controlling the envelope shape. Time Effort controls a $15 \mathrm{~dB}$ range for peak emphasis with Weight Effort adding an additional $3 \mathrm{~dB}$ range onto the peak amplitude.

We found qualitative correlations between Weight Effort and timbre as well as between Weight Effort and pitch [4]. For example, Strong Weight Effort sounds tended to contain more dark tone and dark noise labels, whereas Light Weight Effort sounds had more mid and bright tone labels. While the two Weight Effort qualities share pitch in our correspondence findings (section 
Table 2: P values for significant findings comparing Effort Factors to sound qualities

\begin{tabular}{|c|c|c|c|}
\hline Feature & Effort Factor & P value & Result Summary \\
\hline Amp. Envelope Entropy & Time & $<0.001$ & Sudden sounds tend to contain strong peaks \\
Spectral Flux Entropy & Time & $<0.001$ & Sudden sounds tend to contain peaks of intense change \\
Brightness & Time & $<0.01$ & Sudden sounds tend to be brighter \\
Spectral Centroid (Log Hz) & Time & $<0.01$ & Sudden sounds tend to contain higher frequencies \\
Spectral Flatness & Time & $<0.01$ & Sudden sounds tend to be noisier \\
Spectral Roll-off $(\log \mathrm{Hz})$ & Time & $<0.01$ & Sudden sounds tend to contain more high frequencies \\
Zero-crossing Rate (Log Hz) & Time & 0.0198 & Sudden sounds tend to be noisier \\
Envelope Peak (dB) & Weight & 0.0261 & Strong sounds tend to contain louder peak values \\
\hline
\end{tabular}

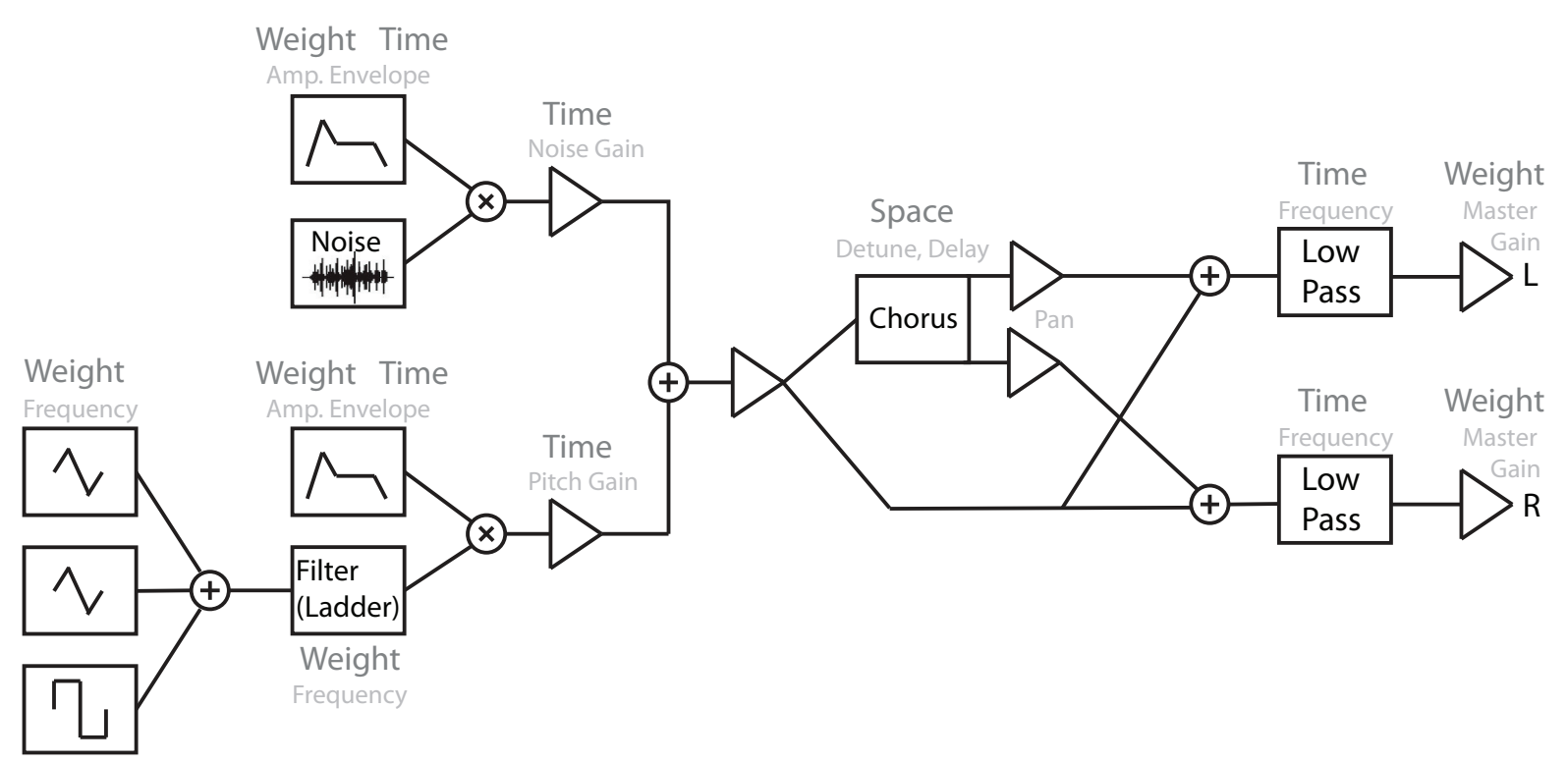

Figure 3: Schematic diagram for movement sonification with Laban Effort Factor (Space, Weight, Time) indicators for parameter control.

3.2), differences exist between pitch and brightness. For example, there is a qualitative difference between a bright tone and a highpitched tone. Brightness may correspond to noise or pitched signals, whereas pitched tones refer mainly to tonal signals (repeating waveforms). In order to differentiate between pitch and brightness (where brightness is already controlled by Time Effort), oscillator frequency and resonant filter frequency are controlled by Weight Effort. Specifically, Weight Effort affects the sound's fundamental frequency, from $130.81 \mathrm{~Hz}$ to $523.25 \mathrm{~Hz}$, representing a two-octave range between Strong and Light Weight sounds. The two-octaves includes portions of all vocal ranges within its scope [22]. Due to the difference in perceived loudness at high and low frequencies as outlined by Fletcher Munson curves [23], Weight Effort slightly adjusts the master gain in order to account for these differences. We use the nominal mixing level of $80 \mathrm{~dB}$ to calculate the difference between fundamental frequency of pitched sounds and the state-variable filter of noise sounds, such that for Strong Weight Effort sounds, the master gain is equivalent to OdBFS and for Light Weight Effort sounds, the master gain is -6dBFS.

Since timbre labels differ for pitch in our correspondence findings, Weight Effort also controls a resonant filter frequency, a
$\operatorname{Moog}^{\mathrm{TM}}$ ladder filter, where the resonant filter alters the timbre of a pitched tone. The range of the resonant frequency is from Eb4 (311.13 hz) to G\#7 (3322.4 hz), based upon formant frequency averages by [24] and [25]. The use of a rectangular wave and two sawtooth waves in the generation of the pitched sound creates enough spectral content for the Moog ${ }^{\mathrm{TM}}$ ladder filter to effectively output identifiably different resonant frequencies.

\subsubsection{Space}

For Space Effort, we found no substantial quantitative nor qualitative correlations between movement and sound qualities. However, in order to test possible perceptual indicators of Space Effort in a future study, we chose to include parametric support for Space Effort based upon 'intuitive' mappings. Spatial placement of sound is a creative dimension of music composition, and spatial metaphors are often used in describing music and our experience of listening to music [26]. In our previous study, we analyzed monophonic sounds bereft of spatial movement. Thus, while Space Effort does not entirely correlate to the spatial domain, we introduce the stereo field and polyphonic sound in our application 
in order to introduce an 'attitude' toward space that the Laban Effort Factor suggests.

For example, chorus is a common effect used to thicken a sound through pitch and timing variations of a split/copied signal. Short delay times $(\leq 25 \mathrm{~ms})$ keeps localization intact due to the precedence effect [27], and just like in audio production applications, additional panning of the effected sound will further spread the sound source image. Space Effort movements Direct and Indirect correspond to the attention a movement pays to the environment (which include spatial indicators), so that a chorus effect naturally underscores sonic spatial indications, including direct sound (doubled with no effect) and indirect sound (spatially diffuse). Space Effort builds a basic Chorus effect by controlling simple detuning, delay, and panning parameters. Indirect Space Effort detunes (half-semitone), delays (25 ms) and pans (50\% right) a signal, whereas Direct Space Effect adds no effect.

The combination of synthesis parameters to Effort Factors is compiled as schematic in Table 3 and sound examples for the Eight Basic Effort Actions is shown in Table 4, which can be heard in [28].

\section{DISCUSSION}

Our sound application generates sounds based upon perceptual links between sound and movement. Individual sounds correspond to individual movements, which serve as basic building blocks of multi-modal stimuli (movement-sounds) and create the possibility for longer, multi-modal combinations of movement and sound. Effenberg [10] discusses the enhanced impact of multi-modal stimuli on motor perception, and Godøy [29] reasons that three motioneffort shapes: Impulsive, Sustained, and Iterative, informs crossmodal perception of sound and movement. The incorporation of peak emphasis into our design characterizes the shape of our sounds in accordance with two of Godøy's Shape descriptions: Impulsive and Sustained [29]. We did not find enough qualitative or quantitative evidence to help us construct the third sound shape, Iterative. We hypothesize that modulating signals are perceptible, and in an effort to further understand how Space Effort may be perceived in future study, we chose Space Effort to control simple frequency and timing modulations (chorus).

We recognize that our findings for peak amplitude emphasis does not include when in time the peak will occur. This problem may be due to that Time Effort Factor does not correlate to our general use of 'time' when describing sound. Specifically, Time Effort does not directly correspond to event timing or sonic duration. Instead, Time Effort describes the urgency of a movement, which can be Sudden or Sustained. Thus, our current solution is to manually align the 'peak' of a movement and the sound (a user parameter controlling peak emphasis location). We may be able to identify when a 'peak' occurs with respect to the movement trajectory by further studying the physical limits of robotic platforms.

We used qualitative results of Weight Effort to help bifurcate the differences between pitch and timbre. While this control stems from a qualitative finding, an exercise from a Laban/Bartenieff Movement Studies (LBMS) training workshop may help underscore the use of formant frequencies in relation to the body. In the LBMS exercise, participants are asked to vocalize vowel formants ( $\bar{u} \bar{o} \bar{a} \bar{e} \bar{i}$ ), focusing on the resonance of the sound within different areas of the body. The vowels resonate from the lower abdomen

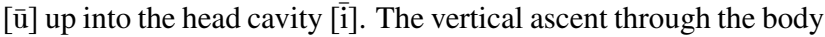
moves due to a shift in Weight Effort, and may help explain quali- tative results distinguishing pitch and timbre. The use of a formant filter in our synthesis application allows sounds to be shaped using frequency irrespective of pitch.

\section{CONCLUSION}

Our synthesized sounds parallel single movement trajectories, currently lasting between one to four seconds. Indeed, the duration of sounds we generate falls within the perceptual timing of Godøy's meso-level timescale, a timing range that suggests the amount of information that may be retained in short-term memory [30]. Generating longer and more complicated, multi-tier movements with controllable movement qualities is an area of ongoing research. Using our application, we could feasibly sequence movements and sounds together to form multi-movement phrases and patterns. The development of longer phrases would then allow for the study of Laban's fourth Effort Factor: Flow, which roughly speaking, describes movement phrasing. Further investigation into longer movement phrases may also be necessary to consider Space Effort quantitatively.

In order to develop a more robust sonificaiton framework, further research into the known physical limits of robotic platforms (i.e. gears and motors) may help deduce sonic duration as well as timing for peak amplitude emphasis. Our proposed framework provides a basis for mobile real-time movement sonification. An upcoming perceptual study using these designed sounds will further unpack related issues, and the study will help document how empirically informed sound design may be used to improve the perception of expressive robotic movement.

\section{ACKNOWLEDGMENT}

The study was made possible in part by the University of Virginia Data Science Institute, Office of the President, and the University of Virginia Office of Graduate and Postdoctoral Affairs. The authors also wish to thank Catherine Maguire, CMA, for movement training in the Laban Effort Factors.

\section{REFERENCES}

[1] A. LaViers, L. Bai, M. Bashiri, G. Heddy, and Y. Sheng, "Abstractions for design-by-humans of heterogeneous behaviors," in Dance Notations and Robot Motion. Springer Tracts in Advanced Robotics (STAR), 2015, pp. 237 - 262.

[2] H. Knight and R. Simmons, "Expressive motion with $\mathrm{x}, \mathrm{y}$ and theta: Laban effort features for mobile robots," in The 23rd IEEE International Symposium on Robot and Human Interactive Communication. IEEE, 2014, pp. 267 - 273.

[3] L. Bai, J. Bellona, L. Dahl, and A. LaViers, "Design of perceptually meaningful quality in robotic motion," in Workshop on Artistically Skilled Robots, IEEE/RSJ International Conference on Intelligent Robots and Systems, 2016.

[4] L. Dahl, J. Bellona, L. Bai, and A. LaViers, "Data-driven design of sound for enhancing the perception of expressive robotic movement," in International Conference on Movement and Computing, 2017.

[5] O. Lartillot and P. Toiviainen, "A matlab toolbox for musical feature extraction from audio," in International Conference on Digital Audio Effects, 2007, pp. 237-244. 
Table 3: Sound controls for Laban's Effort Actions leveraged from [4], as shown in table 2

\begin{tabular}{|c|c|c|c|}
\hline Effort Factor & Parameter & Quality & Quality \\
\hline & & Strong & Light \\
Weight & Peak Envelope Emphasis & $0 \mathrm{dBFS}$ & $-3 \mathrm{dBFS}$ \\
Weight & Overall Amplitude & $0 \mathrm{dBFS}$ & $-6 \mathrm{dBFS}$ \\
Weight & Oscillator Frequency & C3 $(130.81 \mathrm{~Hz})$ & C5 $(523.25 \mathrm{~Hz})$ \\
Weight & Filter Formant Frequency & Eb4 $(311.13 \mathrm{~Hz})$ & G\#7 $(3322.4 \mathrm{~Hz})$ \\
\hline & & Sudden & Sustained \\
Time & Peak Envelope Emphasis & $-3 \mathrm{dBFS} \mathrm{Peak}$ & $-18 \mathrm{dBFS} \mathrm{No} \mathrm{Peak}$ \\
Time & Low-Pass Filter Cutoff & $10000 \mathrm{~Hz}$ & $1000 \mathrm{~Hz}$ \\
Time & Pitch Amplitude & $-12 \mathrm{dBFS}$ & 0dBFS \\
Time & Noise Amplitude & OdBFS & $-12 \mathrm{dBFS}$ \\
\hline & & Direct & Indirect \\
Space & Chorus:Pitch & No Effect & Half-semitone \\
Space & Chorus:Delay & No Effect & $25 \mathrm{~ms}$ \\
Space & Chorus:Pan & No Effect & Right $50 \%$ \\
\hline
\end{tabular}

Table 4: Sound qualities for Eight Basic Effort Actions based upon quantitative sound design

\begin{tabular}{|c|ccc|}
\hline Movement & Tone & Volume & Amp. Envelope \\
\hline Gliding & Bright Tone & Quieter & Sustained \\
Pressing & Dark Tone & Louder & Sustained, Small Peak \\
Floating & Dark Noise & Quieter & Sustained \\
Wringing & Dark Noise & Louder & Sustained, Small Peak \\
Dabbing & Bright Tone & Quieter & Peak Envelope \\
Thrusting & Dark Tone & Louder & Large Peak Envelope \\
Flicking & Bright Noise & Quieter & Peak Envelope \\
Slashing & Bright Noise & Louder & Large Peak Envelope \\
\hline
\end{tabular}

[6] M. M. Wanderley, "Quantitative analysis of non-obvious performer gestures," in Gesture and Sign Language in Humancomputer Interaction, I. Wachsmuth and T. Sowa, Eds. Berlin: Springer Verlag, 2002, pp. 241 - 253.

[7] B. Buck, J. Macritchie, and N. J. Bailey, "The interpretive shaping of embodied musical structure in piano performance." Empirical Musicology Review, vol. 8, no. 2, pp. 92 $-119,2013$.

[8] A. O. Effenberg, "Movement sonification: Effects on perception and action," IEEE MultiMedia, vol. 12, no. 2, pp. 53 - 59, 2005. [Online]. Available: http://ieeexplore.ieee. org/document/1423934/

[9] G. Schmitz, B. Mohammadi, A. Hammer, M. Heldmann, A. Samii, and T. F. Mnte, "Observation of sonified movements engages a basal ganglia frontocortical network." $B M C$ Neuroscience, vol. 14, no. 1, pp. 1 - 11, 2013

[10] A. O. Effenberg, U. Fehse, G. Schmitz, B. Krueger, and H. Mechling, "Movement sonification: Effects on motor learning beyond rhythmic adjustments." Frontiers in Neuroscience, pp. $1-18,2016$.

[11] P. M. Vinken, D. Krger, U. Fehse, G. Schmitz, H. Brock, and A. O. Effenberg, "Auditory coding of human movement kinematics." Multisensory Research, vol. 26, no. 6, pp. 533 552, 2013.

[12] G. Schmitz and A. O. Effenberg, "Perceptual effects of auditory information about own and other movements," in Proceedings of the 18th International Conference on Auditory Display, Atlanta, GA, June 2012.

[13] H. Brock, G. Schmitz, J. Baumann, and A. O. Effenberg", "If motion sounds: Movement sonification based on inertial sensor data," Procedia Engineering, vol. 34, pp. 556 - 561, 2012. [Online]. Available: http://www.sciencedirect.com/ science/article/pii/S1877705812017080

[14] A. LaViers and M. Egerstedt, "Style-based robotic motion," in Proceedings of the 2012 American Control Conference, Montreal, QC, June 2012.

[15] J. Françoise, S. Fdili Alaoui, T. Schiphorst, and F. Bevilacqua, "Vocalizing dance movement for interactive sonification of laban effort factors," in Proceedings of the 2014 conference on Designing interactive systems. ACM Press, 2014, pp. 1079-1082. [Online]. Available: http://dl.acm.org/citation.cfm?doid=2598510.2598582

[16] G. Dubus and R. Bresin, "A systematic review of mapping strategies for the sonification of physical quantities." PLoS $O N E$, vol. 8, no. 12, pp. $1-28,2013$.

[17] J. Wilson-Bokowiec and M. A. Bokowiec, "Kinaesonics: The intertwining relationship of body and sound." Contemporary Music Review, vol. 25, no. 1/2, pp. 47 - 57, 2006.

[18] J. Bellona, "Casting (for kinect, kyma, and processing)," http://jpbellona.com/work/casting/, 2013.

[19] K. Studd and L. Cox, Everybody is a Body. Indianapolis, IN: Dog Ear Publishing, 2013.

[20] R. Laban and F. C. Lawrence, Effort: Economy of Human Movement, 2nd ed. Macdonald and Evans, June 1974.

[21] J. Bellona, "Jpb.mod*, a data modification toolkit," http:// jpbellona.com/work/jpb-mod/.

[22] I. Titze, T. Riede, and T. Mau, "Predicting achievable fundamental frequency ranges in vocalization across species." PLoS Computational Biology, vol. 12, no. 6, pp. 1-13, 2016. 
[23] H. Fletcher and W. A. Munson, "Loudness, its definition, measurement and calculation," Journal of the Acoustical Society of America, vol. 5, pp. 82 - 108, 1933.

[24] J. Catford, A Practical Introduction to Phonetics (Oxford Textbooks in Linguistics), 2nd ed. Oxford University Press, 2002.

[25] G. E. Peterson and H. L. Barney, "Control methods used in a study of the vowels," The Journal of the Acoustical Society of America, vol. 24, no. 2, 1952. [Online]. Available: http://asa.scitation.org/doi/pdf/10.1121/1.1906875

[26] M. L. Johnson and S. Larson, "Something in the way she moves - metaphors of musical motion," Metaphor and symbol, vol. 18, no. 2, pp. 63-84, 2003.

[27] B. Krapalos, M. R. M. Jenkin, and E. Milios, "Auditory perception and spatial (3d) auditory systems," Technical Report CS-2003-07, 2003.

[28] J. Bellona, L. Dahl, L. Bai, and A. LaViers, "Empirically informed sound synthesis application playing laban's eight basic effort actions," YouTube, 2017. [Online]. Available: https://youtu.be/R8NQ8LYfv08

[29] R. I. Godøy, M. Song, K. Nymoen, M. R. Haugen, and A. R. Jensenius, "Exploring sound-motion similarity in musical experience," Journal of New Music Research, vol. 0, no. 0, pp. 1-13, 2016. [Online]. Available: http://dx.doi.org/10.1080/09298215.2016.1184689

[30] R. I. Godøy, "Gestural affordances of musical sound," in $\mathrm{Mu}$ sical gestures: sound, movement, and meaning, R. I. Godøy and M. Leman, Eds. New York: Routledge, 2010, ch. 5, pp. 103-125. 